\title{
Intelligent Aspect based Model for Efficient Sentiment Analysis of User Reviews
}

\author{
Dr. M. Deva Priya ${ }^{1}$ and R. Rithika ${ }^{2}$ \\ ${ }^{1}$ Assoc. Professor, Sri Krishna College of Technology/CSE Department, Coimbatore, Tamil Nadu, India \\ Email: m.devapriya@skct.edu.in \\ ${ }^{2}$ UG Scholar, Sri Krishna College of Technology/CSE Department, Coimbatore, Tamil Nadu, India \\ Email: ritzmaha027@gmail.com
}

\begin{abstract}
Mining online reviews to extract opinion targets and words are substantial tasks in fine-grained opinion mining. The main aim is to mine sensible multi-grain aspects and opinion words from unlabeled reviews. In this paper, Combined Aspect based Sentiment Model (CASM) is propounded to cooperatively mine multi-grain features and opinions. CASM deals with aspects, opinions, sentiment polarity and granularity concurrently. Support Vector Machine-Radial Basis Function kernel (SVM-RBF) classifier is applied to improve CASM by splitting aspects and opinion words. CASM-SVM-RBF deals with two different kinds of aspects and opinions: general and particular aspects, overall opinions and aspect-precise opinions. Candidates with improved confidence are mined as opinion targets or words. The performance is analyzed in terms of Accuracy, Precision, Recall and F-Score for KNN, BPNN, NBC and ME classifiers.

Index Terms: Aspects, Opinions, Sentiments, Support Vector Machine, Radial Basis Function, Support Vector Machine (SVM), Radial Basis Function (RBF)
\end{abstract}

\section{INTRODUCTION}

In recent times, many online shopping sites have come into existence with the speedy increase of e-commerce and online merchants. To provide customer satisfaction, merchants and producers call for customer reviews that include views of the products bought or services demanded. Finally, the customers' opinions on the numerous aspects of entities are aggregated. The aspects of different granularities are to be automatically extracted, followed by identification of related opinions, particularly aspect-based opinions and classification of sentiment polarity.

These reviews subsequently become an intellectual basis of information. This information base is beneficial to customers and producers. Based on the reviews, the customers take decisions whether to buy a product or not. The producer on the other hand uses this information to comprehend and know the likings of customers. It greatly aids them in developing better products, marketing and maintaining consumer relationship. Further, these customer reviews inspire the decisions of other customers. Hence, these reviews play a dominant role in supporting the business organizations to implement their development plans. There are 2 categories of textual data namely, facts and opinions. Web searching and text mining deal with facts. Opinion mining encompasses Natural Language Processing (NLP), computational linguistics and text mining. It involves study of emotions, sentiments and opinions conveyed in text.
A sentiment is a thought, view or attitude that is based on emotion. Opinion mining is otherwise called sentiment analysis. This field is used by organizations or individuals for knowing the overall sentiment related to a specific object like product, person, movie, institution etc., Earlier, with insufficient admittance to user produced opinions, research in this field was insignificant. With the incredible growth of the World Wide Web (WWW), vast amounts of texts including blogs, reviews, etc., have become available for analysis. WWW is the quickest, wide-ranging and effortlessly available medium that supports sentiment analysis.

Nevertheless, finding opinions and observing them on the web is a challenging task as varied sources are available with enormous amount of information. From a human's viewpoint, it is demanding and tedious to find suitable sources, mine relevant sentences, read and process them, and establish them into a serviceable form. Here comes the need for an automated mining and summarizing model. Various models are available in the literature that process some of these tasks, but little work is done in developing models that enable all the operations to be performed concurrently.

In this paper, a Combined Aspect based Sentiment Model (CASM) model is proposed to cooperatively extract multigrain aspects and opinions that is capable of addressing all the tasks. CASM is capable of dealing with the aspects, opinions, sentiment polarity and granularity concurrently. To perform separation of opinion and aspect words, CASMSVM-RBF is proposed, wherein Support Vector MachineRadial Basis Function kernel (SVM-RBF) classifier is applied to improve CASM.

\section{A. Opinion Mining}

Opinion mining is a version of Natural Language Processing (NLP) which is extensively used to track a particular product that the customer is opting. It is also known as sentimental analysis and acts as a platform that builds a system to categorize opinions over a product. Opinion mining can be integrated with Machine Learning (ML) to automate the process, thus forming a system for analyzing sentiments. It helps marketers to:

$\checkmark$ Analyze the reach and success of an advertisement/ launch of a product

$\checkmark$ Regulate a product/service's versions

$\checkmark$ Assess the statistics of pros/cons of a particular product 
For example, a mobile phone may have positive feedbacks considering the camera quality, yet might contain negatives reviews relating to the weight of the phone. This facilitates the vendor to obtain a clear idea of public opinion in contrast to the focus groups or surveys, as the reviews rendered thus are from customers.

\section{Levels of Opinion Mining}

Opinion mining tasks are categorized based on the level at which mining is performed. The different levels include:

$\checkmark$ The document level

$\checkmark$ The sentence level

$\checkmark$ The feature level

$\checkmark$ Document level - Documents are categorized based on sentiments into positive, negative and neutral polarity. It is assumed that the document focuses only on a single aspect of the object. Opinions from the opinion holder are also included.

$\checkmark$ Sentence level - At this level, data is classified into objective, subjective or opinionated text from the corpus and subjective or opinionated sentences are identified. As a result, the sentences in the document are classified into positive, negative and neutral polarities.

$\checkmark$ Feature level - There are a number of tasks involved in this level which are listed below:

$\checkmark$ Task 1: It recognizes and extracts object features commented on review/text

$\checkmark$ Task 2: Deals with inferring the opinions as positive, negative or neutral

$\checkmark$ Task 3: Groups similar features and produces feature based opinion summary of multiple reviews/text

These tasks are performed if the features and the synonyms are unknown. If the features are known, and the synonyms are unknown, then all the tasks are to be performed though the third step is easier. The new features that are discovered are mapped with the existing features. If they match, it is mandatory to perform task 2 .

\section{B. Challenges in Opinion Mining}

Following are some of the challenges of opinion mining:

$\checkmark$ A word can sound positive and negative in various circumstances. The words render completely different meanings in each of the scenarios. For instance, with respect to a product review of a mobile, the word "long" gives a positive outlook when it is described for battery performance but gives a negative opinion when it comes to the booting speed. Therefore, the proposed system must be able to identify the category of opinions.

$\checkmark$ It is to be noticed that the choice of expression varies for a large span of people. Small changes between two text fragments do not hinder the contextual meaning. But, as far as opinion mining is concerned, basic conjunctions like 'and', 'but' and words that offer remarks like 'not' and 'very' must be scrutinized.
Some views might be contradictory in the sense, the reviews can include both positive and negative statements which can be best suited for analysis when each sentence is analyzed separately. Due to the informal medium that is addressed, the reviews are complex with a variety of opinions in a single sentence. Like the remark which states "The overall performance is very good except for the longevity of the battery charge which is not encouraged."

\section{Sentence-Level Sentiment Analysis}

Web opinion mining revolves around sentimental classification at the document-level. Document-level classification being too coarse for a majority of the applications, analysis is applied at the sentence-level. Classifying sentences based on objective or subjective speech is the main agenda of this analysis, and not extracting subjective sentences in news articles.

\section{RELATED WORK}

Opinion target and word extraction are the predominant tasks involved in opinion mining. Much work is done on them [1-5]. Opinion mining is divided into two types: sentence-level and corpus level based on their extraction aims. Hu \& Liu (2004) [1] have used nearest-neighbor rules to recognize opinion relations that exist among words. Using a bootstrapping process, frequent and explicit product features are extracted. Using co-occurrence information or nearest-neighbor rules can never help in precisely detecting opinion relations among words.

Popescu \& Etzioni (2007) [4] have used syntax related information to mine opinion targets and have developed syntactic patterns that identify opinion associations amid words. They have shown that their scheme is better than that of $\mathrm{Hu} \& \mathrm{Liu}$ (2004) [1, 6]. Identifying and computing opinion associations among words are the key components. Wang \& Wang (2008) [5] have used the co-occurrence frequency of opinion targets and words to specify associations.

In Sequence-labelling problems, extraction at sentencelevel deals with extracting opinion targets or words by identifying the opinion targets or expressions [7-9]. Naturally, appropriate words are considered as features that specify opinion targets/words in sentences.

Ma \& Wan et al (2010) [9] have also used CRF to mine opinion targets from reviews. These methods make use of labelled data for model training. In case of insufficient labelled training data, data is taken from varied domains in contrast to the available text leading to reduction of the extraction performance. They have propounded a mechanism that depends on transfer learning to ease cross domain mining of opinion targets/words. This mechanism uses labelled data from out-domains as the mining performance wholly depends on the significance of indomain and out-domain. Further, more work is done on corpus-level mining. Opinion target/word in sentences is not identified, but a set of opinion targets is mined or a sentiment word lexicon is generated from texts. Much of the earlier schemes have used a cooperative unsupervised mining framework. 
Further, traditional sequence labelling models such as Conditional Random Fields (CRFs) [10] and Hidden Markov Model (HMM) [11] are also used for extraction. Jin et al (2009) [11] have propounded a lexicalized HMM model to perform opinion mining.

Various authors have used topic modelling to detect inherent topics and sentiment words [12-15]. The main goal is not to mine an opinion target or word lexicon, but cluster words into conforming features in reviews. These mechanisms have used frequency statistics and phrase identification to identify right opinion targets/words. They have emphasized on clustering words into consistent topics or features. Mining opinion targets and words from online reviews are significant tasks in opinion mining, which involve identifying opinion associations among words.

Qiu et al (2009) [16] \& Qiu et al (2011) [17] have propounded Double Propagation that involves syntactic associations amongst words to iteratively enlarge sentiment words and opinion targets. The chief restriction is that the patterns are based on dependence parsing tree and hence do not deal with all opinion associations. Liu et al (2012) [18] have dealt with opinion target mining based on unsupervised WAM that captures opinion associations in sentences. Opinion targets are mined in a regular random walk framework making it efficient for mining opinion targets. However, they have not dealt with demonstrating the efficiency of the WAMon opinion word mining. Kaur \& Gupta (2013) [19] have stated that the machine must be able to infer the NLP techniques well before sentimental analysis phase. This technique is tested against languages like English, Hindi with the help of senti-wordnet to estimate the polarity of the sentences. To accomplish this, the machine is required to fathom NLP through training. Hasan et al (2014) [20] have performed sentimental analysis with Bangla text. In spite of acquiring senti-wordnet, the experiment displays difficulty in analysis as the texts are completely in Bangla and not English.

Asghar \& Khan (2014) [21] have proposed a sentimental analysis which classifies subjective and objective sentences from collection of reviews. They have used senti-wordnet to predict the score by determining the polarity of the sentence. Ahmed et al (2015) [22] have surveyed on tool enhancement methods and tools available for analysis. Though the analysis of the applications is done, the comparative approaches for these applications are not accomplished. Sri \& Ajitha (2016) [23] have applied sentiment classification to conduct a survey on product reviews. Frequent terms are found easily by using a new technique named semantic orientation. With time complexity and accuracy as parameters, a comparative study between existing and proposed solutions is conducted which shows that semantic orientation is the best.

Zhang et al (2017) [24] have stated that opinions rendered on various aspects of entities described in larger scale online reviews require automatic extraction of granularities, recognizing opinions that associate with the granularity and categorizing sentiments based on polarity. Although various models are proposed to process few of the tasks, a model to simultaneously perform the task is uncommon. This model focuses on separating polarity, opinion and model aspects separately. To do so, Joint Aspect-Based Sentiment Topic with Maximum Entropy (JABST-ME) classifier is applied. Dubey et al (2018) [25] have suggested an item based collaborative filtering system to overcome the demerits of the conventional collaborative algorithm. The authors have proposed an improved recommender system. The system involves a record of sentiment scores which is determined by calculating the positive reviews. The scores are thus used in a filtering system which differentiates the overall negative user opinions from positive ones so as to improve the recommendation of the product.

Al-Ayyoub et al (2019) [26] have dealt with the online social networks that have paved the way for many domains including analysis of networks and contents to derive information. Sentimental analysis being one of the advancement is involved in extracting sentiments conveyed in the content. Though sentimental analysis has its own history, it has been in use from the recent decade as this allows users to interact over the network and post their views and opinions. Gopi et al (2020) [27] have conducted a survey from which it is inferred that some of the social networking platforms like Facebook offer users an option to express their opinions through emojis. Classifying opinions into different categories is termed as opinion mining, whereas defining a score for these opinions falls under sentimental analysis. The opinions are scored with numbers ranging from -5 to 25 . To prove the theory, movie review data from twitter from the years 2013 to 2020 is used. Sankar et al (2020) [28] have proposed a technique for sentiment classification. To obtain optimal words, Machine Learning (ML) based techniques offer better results. The resulted features are then fed into a Neural Network (NN) for further processing. The approach is evaluated for the datasets like rotten tomatoes. This system is tested over an android application framework on smart phone reviews without the aid of cloud or server side Application Programming Interface (API) to offer effective results.

\section{TeXt Classification Mechanisms}

In this section, some of the renowned text classification schemes are discussed.

\section{A. K-Nearest Neighbor (KNN) Algorithm}

$\mathrm{K}-\mathrm{Nearest}$ Neighbor (KNN) is simple to understand and implement. It is based on statistics and not parameters. It makes decisions based on the trained data. KNN guesses whether the data is present in a feature space or not. Gene array vector is taken as input. Similarity measurement using different measures such as Euclidean, Minkowski and Manhattan are computed and the KNN classifier is trained using the selected features.

KNN depends on a distance function for pairs of data samples such as the Euclidean distance and Pearson's correlation of their gene expression profiles. The data samples are classified based on the class memberships of their ' $\mathrm{K}$ ' nearest neighbors. Many researchers prefer KNN due to its simplicity and its better performance. The classifier defines non-linear decision limits i.e. KNN improves the performance when the sample has insignificant non-linear variations around the decision margin result. 
Given an input gene array vector, the classifier extracts the reference set of ' $K$ ' nearest vectors based on the similarity and decides the label of input vector based on the classifier. Pearson's coefficient correlation, Euclidean, Manhattan and Minkowski distances represent the similarity measures. For an input ' $x$ ' and a reference set,

$$
\mathrm{M}=\left\{\mathrm{m}_{1}, \mathrm{~m}_{2}, \ldots \ldots \ldots \mathrm{m}_{\mathrm{N}}\right\}
$$

The probability that ' $\mathrm{x}$ ' can fit into a class is given by:

$$
\mathrm{P}\left(\mathrm{x}, \mathrm{c}_{\mathrm{j}}\right)=\sum_{\mathrm{m}_{\mathrm{i}} \in \mathrm{KNN}} \mathrm{S}\left(\mathrm{x}, \mathrm{m}_{\mathrm{i}}\right) \mathrm{P}\left(\mathrm{m}_{\mathrm{i}}, \mathrm{c}_{\mathrm{j}}\right)-\mathrm{b}_{\mathrm{j}}
$$

where,

$\mathrm{S}\left(\mathrm{x}, \mathrm{m}_{\mathrm{i}}\right)-$ Similarity amid ' $\mathrm{x}$ ' and ' $\mathrm{m}_{\mathrm{i}}$ '

$b_{j}-$ Bias term

\section{B. Back-Propagation Neural Networks (BPNN)}

Back-Propagation Neural Networks (BPNN) is generally used in training Deep Neural Networks (DNNs) to evaluate the error influence of each neuron for a set of data that is handled [29]. It is an unusual case of automatic differentiation. It is commonly used in adjusting the neuron weights by finding the gradient loss function. This mechanism is also known as backward propagation of errors, as the errors are computed in the output layer and sent back. It demands a known, preferred output for every input and is considered as a supervised learning method.

Back-Propagation is the essence of neural net training. It is the act of streamlining the weights of a neural net with the help of error rate acquired in the former iteration. Appropriate tuning of the weights guarantees lesser error rates making the model dependable by expanding its speculation. The algorithm searches for the reduced value of error function in the weight space by utilizing an approach known as the delta rule or gradient descent. The weights which limit the error function are considered as solutions for the learning problem.

BPNN is capable of creating complex decision boundaries/limits in the feature space. Even an attempt has been made using Multi-Layer Perceptron (MLP) networks trained with backpropagation, Radial Basis Function (RBF) networks and high-order polynomial networks which strongly recommend that a BPNN under suitable conditions can estimate Bayesian posterior probabilities at its outputs. This is critical on the grounds that a Bayesian classifier gives the finest performance with reduced error rate for a feature data distribution. It is not possible to predict the performance of BPNN similar to other non-parametric ways. Furthermore, some parameters like number of training samples and hidden nodes, and the learning rate are to be chosen.

\section{Nä̈ve Bayesian Classifier (NBC)}

Naïve Bayes Classifier (NBC) is the most common classifier for text arrangement. It also serves as the basic criteria for redefining a classifier. For instance, an apple is associated with its features, that is, its color and shape. Based on the likelihood of these organic products, the classifiers are able to differentiate products based on the shading in their color, width and roundness. A demonstration of contingent likelihood is made using a vector $d=\left(d_{1}, \ldots ., d\right)$, with the probability of $p\left(a_{k} \mid d_{1}, \ldots ., d\right)$ for ' $\mathrm{k}$ ' conceivable classes or results ' $\mathrm{a}_{\mathrm{k}}$ '. The classifier defined as,

$$
\frac{p\left(a_{k} \mid d\right)=p\left(a_{k}\right) p\left(d \mid a_{k}\right)}{p(d)}
$$

where,

$$
\text { Posterior }=\frac{\text { Prior } * \text { Likelihood }}{\text { Evidence }}
$$

\section{Maximum Entropy (ME)}

The Maximum Entropy (ME) belongs to the class of exponential models as it is a probabilistic classifier. ME unlike Naive Bayes Classifier (NBC) does not limit the repetition of the highlights. The MaxEnt function based on the principle of ME focuses on choosing the model with high entropy values from a pool of models that match the prepared information. The content order issues can be segregated under various aspects using ME classifier. A few issues which may occur frequently include point characterization, dialect location and supposition examination.

The parameters of a chosen model have to be assessed prior to further execution for which, $\mathrm{ME}$ requires a considerable amount of time to prepare and process the underlying goal, while NBC does not.

The first step is to look into the following arrangement $\left(\mathrm{x}_{\mathrm{i}}, \mathrm{y}_{\mathrm{i}}\right)$ wherein ' $\mathrm{x}_{\mathrm{i}}$ ' stands for the logical data corresponding to the record (meagre cluster) and ' $y_{i}$ ' is it's respective class. The second step focuses on outlining the preparation test approximating to its exact likelihood:

$$
\tilde{\mathrm{q}}(\mathrm{x}, \mathrm{y})=\frac{1}{\mathrm{~m}} \times \mathrm{n}
$$

Where,

$\mathrm{m}$ - Size of the training data

$\mathrm{n}$ - number of times $(\mathrm{x}, \mathrm{y})$ seen in sample

The indicator function is given by,

$f_{j}(x, y)= \begin{cases}1, & \text { if } y=d_{i} \text { and } x \text { contain } v_{k} \\ 0, & \text { otherwise }\end{cases}$

The probability of class is derived as:

$\mathrm{p}(\mathrm{d} \mid \mathrm{e}, \vec{\lambda})=\frac{\exp \left[\sum_{\mathrm{i}} \lambda_{\mathrm{i}} \mathrm{f}_{\mathrm{i}}(\mathrm{d}, \mathrm{e})\right]}{\sum_{\mathrm{d}^{\prime}} \exp \left[\sum_{\mathrm{i}} \lambda_{\mathrm{i}} \mathrm{f}_{\mathrm{i}}\left(\mathrm{d}^{\prime}, \mathrm{e}\right)\right]}$

Where,

d - Class of the model

e - Data point

$\lambda$ - Weight vector.

ME poses to be much efficient with respect to NBC over the following reasons:

- Accurate classification in contrast to NBC

- Portrays consistency and sign of improvement

- Proves to be efficient when dealing with a large volume of data

- Easy processing of data irrespective of the differences in formats 


\section{E. Support Vector Machine (SVM)}

Support Vector Machine (SVM) [30] is a widely used classifier. They are particularly helpful for predicting numerical data, classification and pattern matching techniques. They consider boundaries between data points, focusing on the decision boundary that isolates the data points into classes (or is the most generalizable). The main aim is to have the decision boundary between the points as extensive as possible. This expands the distance between data point and the boundary line.

It is a supervised training model related to learning algorithms for analyzing data and recognizing data patterns. The choice of kernel parameters involved in the training process has an influence on the classification accuracy. Feature selection also plays a major role in classification. It is used in pattern recognition [31], bioinformatics [32] and text categorization [33]. SVM categorizes data by finding a collection of support vectors that belong to a class of training inputs that define a hyperplane in the feature space. It focusses on fitting the hyperplane to the training data by using a kernel function. These functions can be chosen during training to identify support vectors along the function limits.

SVM includes kernels like the linear kernel, polynomial kernel and the RBF kernel, out of which the RBF kernel is the most popular. A function ' $y$ ' that maps a single vector to a higher dimensional vector, approximating the kernel is given below.

$$
\mathrm{y}(\mathrm{x}) \mathrm{y}\left(\mathrm{x}^{\prime}\right) \approx \varphi(\mathrm{x}) \varphi\left(\mathrm{x}^{\prime}\right)=\mathrm{h}\left(\mathrm{x}, \mathrm{x}^{\prime}\right)
$$

Where,

' $\Psi$ 'is the implicit mapping embedded in RBF kernel.

The general function of SVM classifier is given by,

$$
f(z)=\sum_{i=1}^{n} a_{i} h\left(z, z_{i}\right)+c
$$

Where, the feature vector is defined as,

$$
\mathrm{h}\left(\mathrm{z}, \mathrm{z}^{\prime}\right)=\exp \left[-\gamma\left\|\mathrm{z}-\mathrm{z}^{\prime}\right\|^{2}\right]
$$

where,

$\sigma$ - Free parameter

$$
\gamma=\frac{1}{2 \sigma}
$$

\section{Proposed SYSTEM}

In the present system, the opinions are categorized into positive and negative types. Those with neutral opinions are not categorized precisely. Sentiment scores are computed using Senti-WordNet or WordNet tools which include scoring functions to measure the positive/ negative scores proficiently when compared to the neutral score. The proposed system capable of dealing with neutral opinions and a scoring function is designed to offer a better scoring. The proposed system includes the following phases namely, data extraction, opinion extraction and sentiment evaluation.

An aspect term called feature indicates a particular attribute or component of an entity (product) which is a noun or noun phrase. For example, "voice" is an aspect in a review related to audio $\mathrm{CD}$ review. To accumulate opinions on features of objects in online reviews, it is significant to mine aspects of diverse granularities, recognize related opinions, particularly aspect-specific opinions and compute sentiment polarities. The propounded mechanism includes the ensuing steps. In the text mining stage, stop words and symbols are removed. Feature extraction is carried out using stemming, finding Term Frequency (TF) and using Bag of Words (BoW) (Figure 1).

\section{A. Data Collection}

Data is collected from user reviews in blogs, forums and social networks that are not structured properly. It may be conveyed in diverse ways including various words, vernaculars and framework, thus making human analysis complex. Text analytics and NLP ease the process of extraction and classification.

\section{B. Pre-processing}

In this phase, the data is pre-processed to eliminate noise and reduce dimensions. Verbs, nouns and non-verbs in the sentences are identified. The non-textual and inappropriate details are removed from the mined data. For example: common words like "computer" and "touchpad" are identified as aspects. To be specific, "computer" is recognized as a common aspect, while "touchpad" is identified as a particular aspect. It involves the following steps - Tokenization, Stop Word Removal, Stemming, Term Frequency-Inverse Document Frequency (TF-IDF) based feature extraction. 


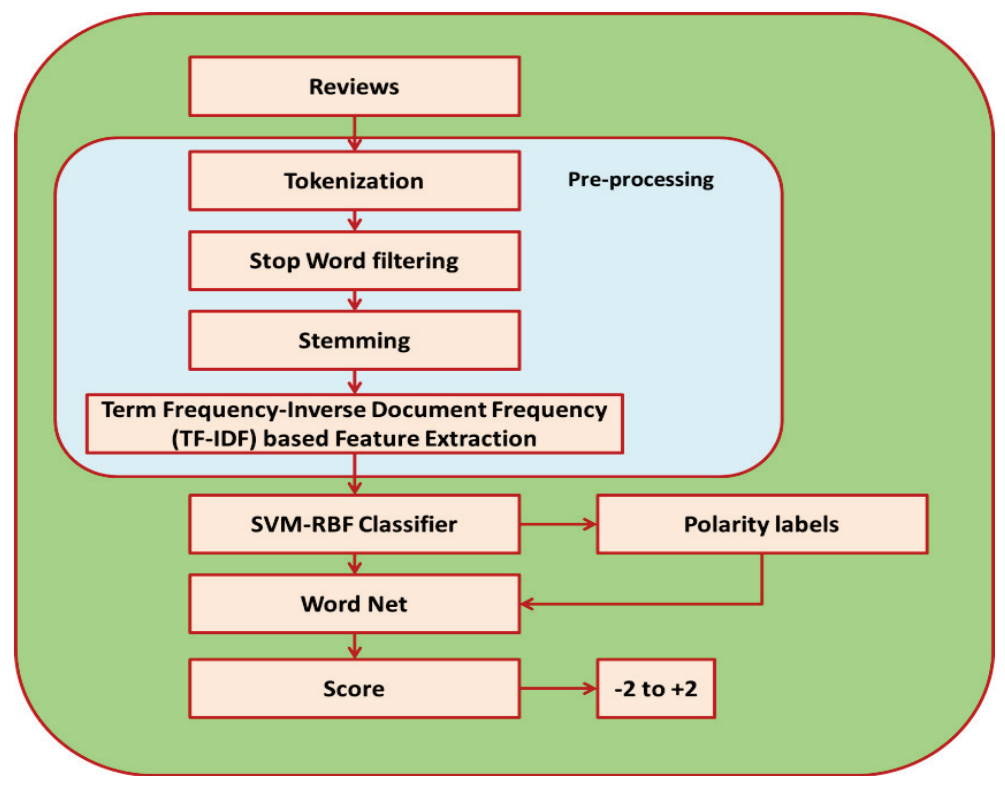

Figure 1. Proposed System

\section{Tokenization}

Tokenization deals with splitting strings into tokens which include words, keywords, phrases, symbols etc., Symbols like punctuation marks are cast-off and meaningful keywords are recognized. In short, it is the process of delineating and categorizing sections of a string. The words in a sentence are explored and consistency is established.

\section{Stop Word Removal}

Stop words include the most frequently used words like 'a, the, an, in'. The search engine ignores them while searching and extracting them based on the query. Removing them reduces the processing time. Stop words are removed before processing of text in natural language. The most common stop words that support text mining are prepositions, articles and pro-nouns.

\section{Stemming}

Stemming is the process of condensing a word to its word stem that relates to the prefix, the suffix or the root word known as lemma. It is also a pivotal component in Natural Language Understanding (NLU) and NLP. Stemming coupled with AI is extensively used for information extraction from big data sources. Interestingly enough, they also serve as a part of the search engine querry for quicker retrieval of data.

\section{Term Frequency-Inverse Document Frequency (TF-IDF) based Feature Extraction}

In this phase, the information with worthy reviews is considered. It is the product of the frequencies of the word considered (TF) and the word in whole corpus (IDF) [34].

$$
\begin{aligned}
& w_{i}=F_{i, j} * \log \left(\frac{N}{N_{i}}\right) \\
& W_{i}-\text { Weight of a term 'i' } \\
& F_{i, j} \text { - Frequency of term 'i' in sample 'j' } \\
& N \text { - Number of samples in the corpus } \\
& N_{i} \text { - Number of samples with 'i' }
\end{aligned}
$$

Adaptive words like 'good', 'bad', 'very', 'not', and conjunctions like 'and, 'but' are identified. Adjectives are to be identified so that the product can be rated correctly. The customers would have rated one aspect positively, but another negatively. The ratings of both the features are to be taken into account while deciding the quality of the product. Combined Aspect based Sentiment Model (CASM) model is propounded to cooperatively mine multi-grain aspects and opinions which focus on all the tasks. CASM is capable of dealing with feature, opinion, sentiment polarity and granularity concurrently.

To isolate opinion and aspects, Combined Aspect based Sentiment Model with Support Vector Machine-Radial Basis Function kernel (CASM-SVM-RBF) is proposed, in which SVM-RBF classifier is applied to improve CASM. SVM-RBF classifier is applied to isolate aspect and opinion words. The goal is to mine multi-grain aspect and opinion words from unlabeled reviews. CASM-SVM-RBF is capable of dealing with different types of aspects and opinions like common and specific aspects, common and aspect-precise opinions.

\section{SVM-RBF Classification}

The adjoining words in the user reviews are taken into account and the aspects are classified into either 'Positive' or 'Negative'. In the proposed scheme, SVM-RBF is used for classification. It is seen that SVM-RBF does effective classification in contrast to $\mathrm{KNN}, \mathrm{BPNN}, \mathrm{NBC}$ and $\mathrm{ME}$ classifiers respectively.

\section{WordNet}

WordNet is a huge lexical database of English. Various viewpoints of a word including nouns, adjectives and adverbs and verbs are collected as cognitive synonyms (synsets), where each has its own structure. To compute the scores of reviews, the dictionary is loaded. By associating the reviews with the dictionary, the scores are anticipated. By involving Senti-WordNet, polarity can be computed. Table 1 shows the opinions and polarities. 
TABLE I.

CLASSIFYING OPINIONS AND POLARITY

\begin{tabular}{|c|c|c|}
\hline Aspect & Opinion & Polarity \\
\hline Mobile screen & Big & Positive \\
\hline Battery & Not lasting & Positive \\
\hline Generator & More noise & Negative \\
\hline
\end{tabular}

A model is built using SVM-RBF and precision is expected. The scores of the reviews are to be considered. The scores are taken as 1 for positive, -1 for negative and 0 for neutral reviews. Nevertheless, a review can include 2 or more sentiments. To predict the scores, a WordNet vocabulary dictionary which includes positive and negative words is stacked. The sentiment reviews which are cast-off in a record are stacked. By differentiating the reviews and the vocabulary word reference, reviews carrying positive and negative views are efficiently observed and scoring is carried out. Positive and negative reviews along with neutral reviews are considered. A scale of -2 to +2 limit is considered to envisage the score, where the scale signifies the values shown in Table 2.

TABLE II.

SCALE FOR SCORES

\begin{tabular}{|c|c|}
\hline Score & Prediction \\
\hline-2 & Awful \\
\hline-1 & Poor \\
\hline 0 & Fair \\
\hline 1 & Good \\
\hline 2 & Excellent \\
\hline
\end{tabular}

\section{IMPLEMENTATION}

The performance of the model is evaluated for reviews related to electronic devices and restaurants, both qualitatively and quantitatively. It is seen that the proposed model outperforms all the benchmarked schemes taken into consideration. The proposed CASM-SVM-RBF offers better accuracy and involves less time period in contrast to the existing methods and algorithms. The screenshots show the steps involved in classifying user reviews (Figure 2 to Figure 8).

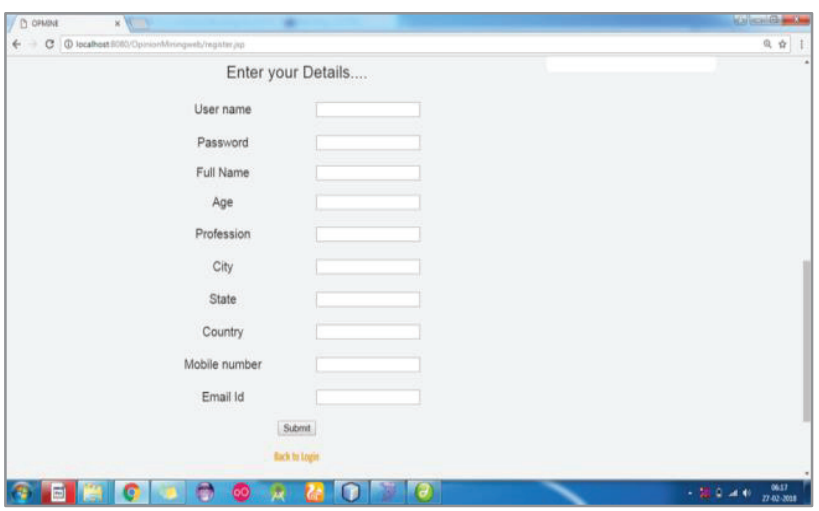

Figure 2. Homepage

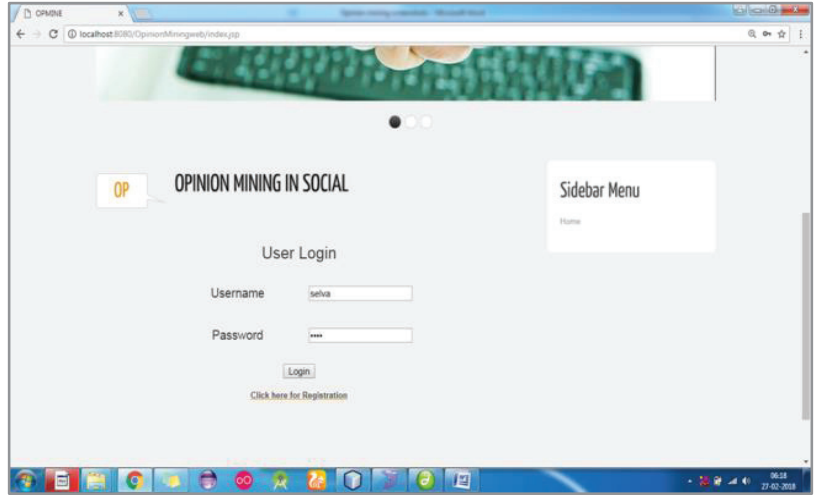

Figure 3. Register and $\log$ in form

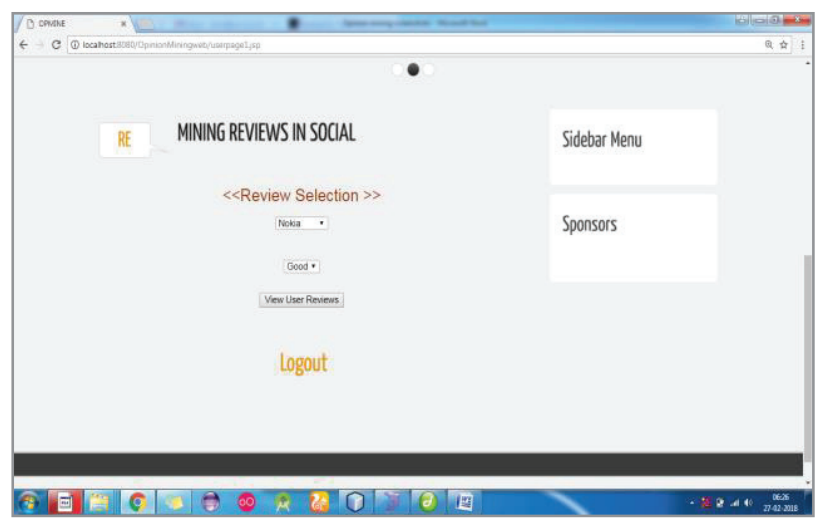

Figure 4. Mining Reviews

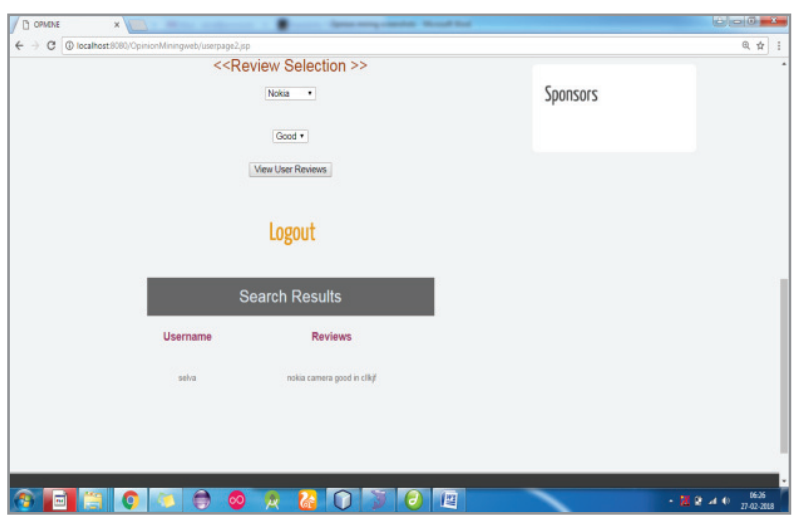

Figure 5. Customer Review Selection

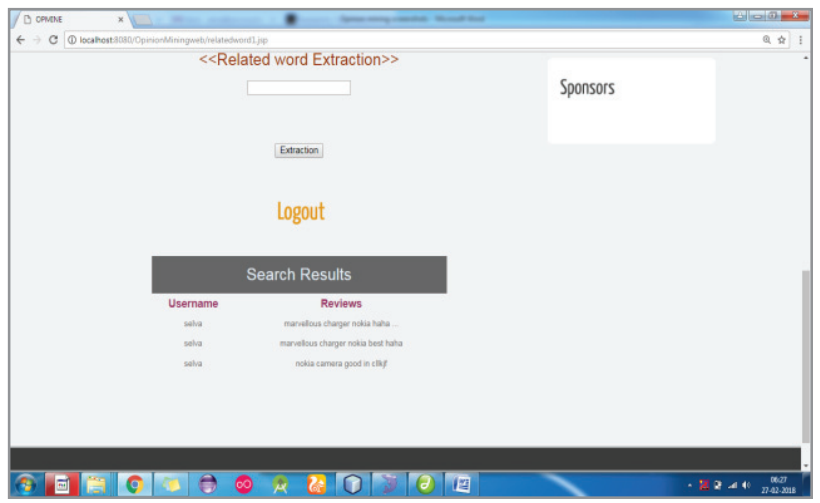

Figure 6. Customer Reviews Search Results 


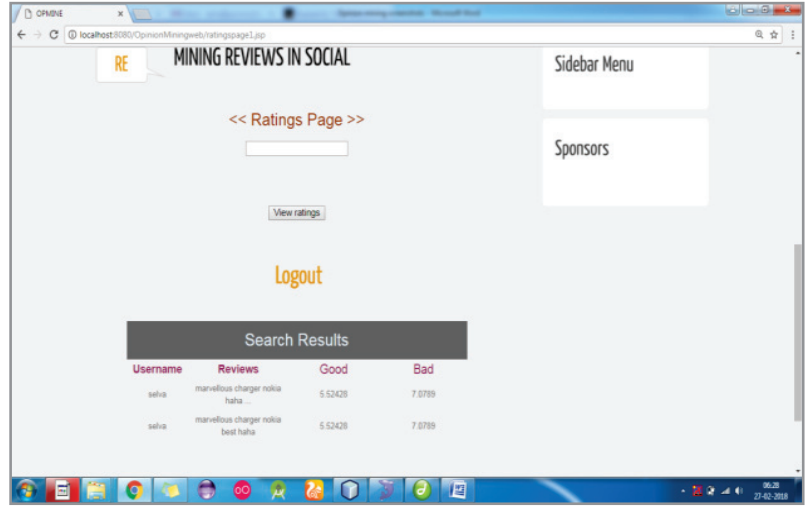

Figure 7. Customer Reviews Search Results Positive \& Negative Ranges

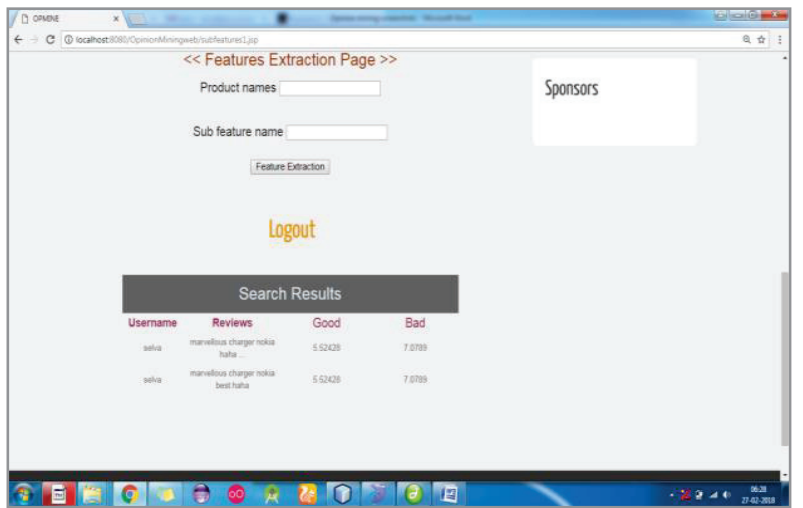

Figure 8. Product based Positive \& Negative Values Calculation

\section{ReSUltS AND DisCUSSION}

A total of 1892 sentences are taken, and spam and recurrent views shown as emotions are removed. SVM-RBF classifier is used and polarity is computed. Once opinion is mined, around 202 sentences are found unique. Using WordNet, scores are found.

The analysis involves sentence based sentiment classification. Hence, a lexicon containing positive and negative words is prepared. The neutral scores are also understood. The lexicon dictionary is improved to compute the score for neutral sentences also. The grades are in the range -2 to +2 . The performance is measured in terms of Accuracy, Precision Recall and F-score.

Accuracy: It is the percentage of predictions that are estimated correctly during the process of opinion mining. It is the ratio of number of predictions that confirm the existence of opinions to the number of predictions imposed for detecting opinions derived from the twitter dataset.

$$
\text { Accuracy }=\frac{\mathrm{TP}+\mathrm{TN}}{\mathrm{TP}+\mathrm{TN}+\mathrm{FP}+\mathrm{FN}}
$$

where,

TN - Correct predictions of an occurrence as negative

$\mathrm{FN}$ - Incorrect predictions of an occurrence as positive

FP - Incorrect predictions of an occurrence as negative

TP - Correct predictions of an occurrence as positive

Precision: It is the percentage of reviews from which opinion is accurately classified. It is the ratio of the number of opinions detected correctly to the number of reviews considered in the opinion mining process.

Precision $=\frac{\mathrm{TP}}{\mathrm{TP}+\mathrm{FP}}$

Recall: It is the percentage of opinion mining that is predicted correctly during the process of opinion mining.

Recall $=\frac{\mathrm{TP}}{\mathrm{TP}+\mathrm{FN}}$

F-score: It is the weighted average computed between the factor of precision and recall values determined during the process of opinion mining.

$$
\begin{aligned}
& \mathrm{F}-\text { score }=2 \times \frac{1}{\frac{1}{\text { Recall }}+\frac{1}{\text { Precision }}} \\
& F-\text { score }=2 \times \frac{\text { Precision } \times \text { Recall }}{\text { Precision }+ \text { Recall }}
\end{aligned}
$$

From Figure 9, it is evident that the SVM RBF offers $31 \%, 21 \%, 16 \%$ and $5 \%$ better accuracy in contrast to $\mathrm{KNN}$, $\mathrm{BPNN}, \mathrm{NBC}$ and ME classifiers respectively.

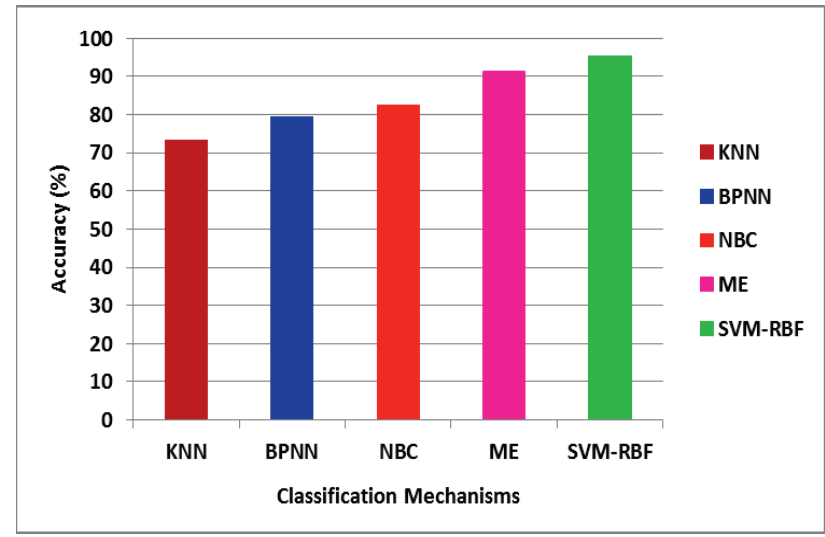

Figure 9. Accuracy

From Figure 10, it is clear that SVM RBF offers $28 \%$, $22 \%, 18 \%$ and $4 \%$ better Precision in contrast to $\mathrm{KNN}$, BPNN, NBC and ME classifiers respectively.

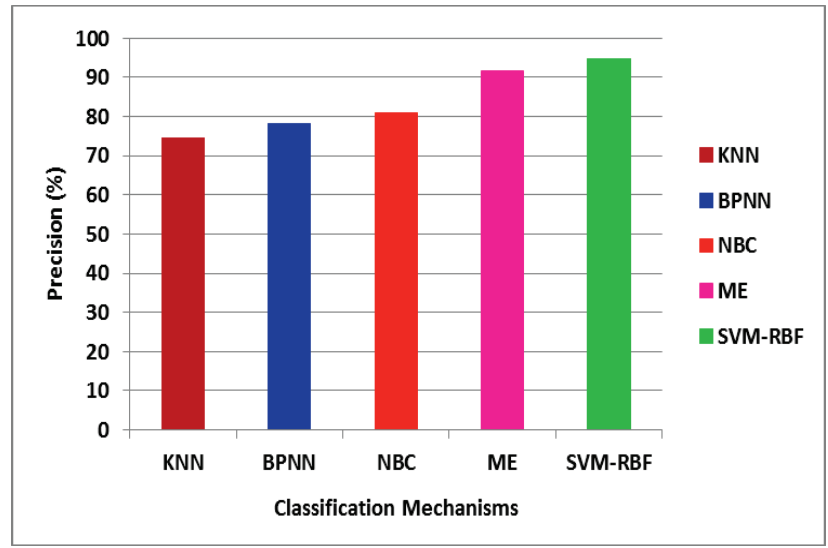

Figure 10. Precision 
From Figure 11, it is clear that SVM_RBF offers 30\%, $23 \%, 17 \%$ and $3 \%$ better Recall in contrast to KNN, BPNN, NBC and ME classifiers respectively.

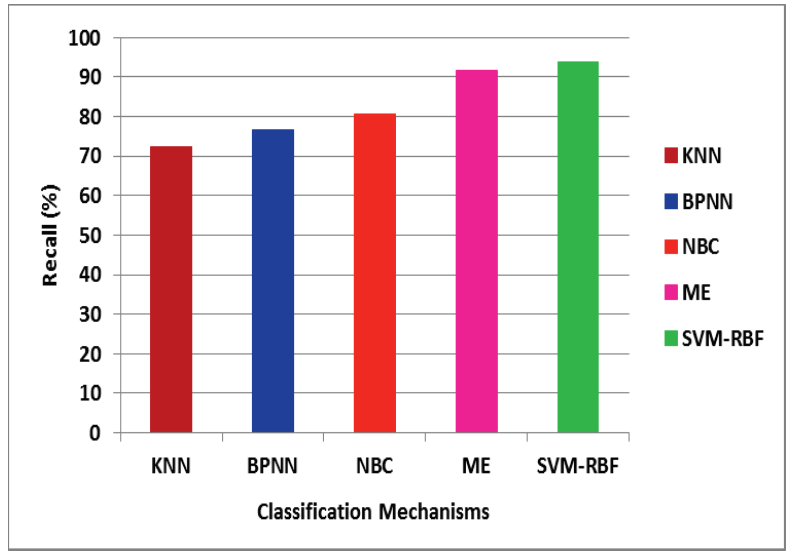

Figure 11. Recall

From Figure 12, it is clear that SVM RBF offers $29 \%$, $27 \%, 14 \%$ and $4 \%$ better $\mathrm{F}$-Score in contrast to $\mathrm{KNN}$, $\mathrm{BPNN}, \mathrm{NBC}$ and $\mathrm{ME}$ classifiers respectively.

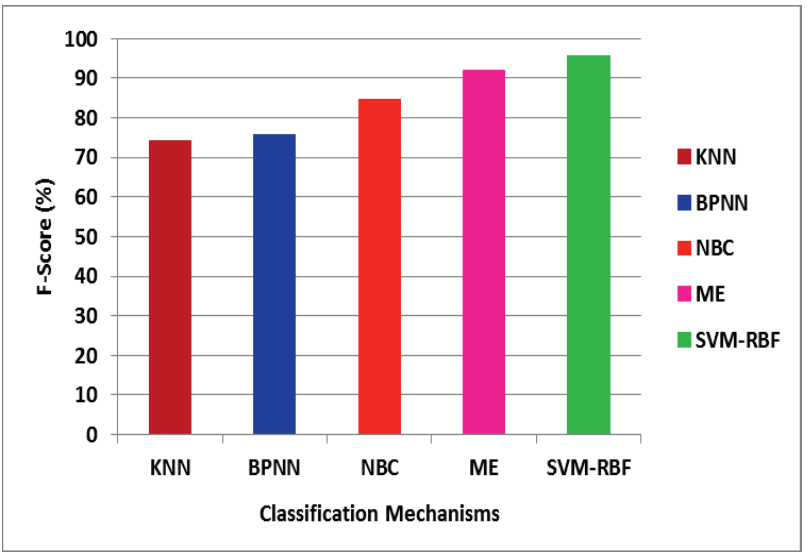

Figure 12. F-Score

\section{CONCLUSIONS}

In this paper, Combined Aspect based Sentiment Model (CASM) is improved by using Support Vector MachineRadial Basis Function kernel (SVM-RBF) classifier. Opinion and aspect words are mined using the CASM and model, wherein SVM-RBF classifier is applied to extend CASM. Multi-grain aspect and opinion words from unlabeled online reviews are extracted. It is seen that SVMRBF classifier offers better results in contrast to $\mathrm{KNN}$, BPNN, NBC and ME classifiers in terms of Accuracy, Precision, Recall and F-Score.

\section{REFERENCES}

[1] Hu, M., \& Liu, B., "Mining and summarizing customer reviews", $10^{\text {th }}$ ACM SIGKDD International Conference on Knowledge discovery and data mining, pp. 168-177, 2004.

[2] Popescu, A.-M., \& Etzioni, O., "Extracting product features and opinions from reviews", Conf. Human Lang. Technol. Empirical Methods Natural Lang. Process., pp. 339 - 346, Vancouver, BC, Canada, 2005.
[3] Ding, X., Liu, B., \& Yu, P. S., "A holistic lexicon-based approach to opinion mining", ACM International Conference on Web Search and Data Mining, pp. 231-240, 2008.

[4] Popescu, A. M., \& Etzioni, O., "Extracting product features and opinions from reviews", Natural language processing and text mining, pp. 9-28, Springer, London, 2007.

[5] Wang, B., \& Wang, H., "Bootstrapping both product features and opinion words from chinese customer reviews with cross-inducing", $3^{\text {rd }}$ International Joint Conference on Natural Language Processing, vol. I, 2008.

[6] Hu, M., \& Liu, B., "Mining opinion features in customer reviews", AAAI, vol. 4, no. 4, pp. 755-760, 2004.

[7] Wu, Y., Zhang, Q., Huang, X., \& Wu, L., "Phrase dependency parsing for opinion mining", Conference on Empirical Methods in Natural Language Processing, Association for Computational Linguistics, vol. 3, pp. 15331541, 2009.

[8] Zhang, Q., Wu, Y., Li, T., Ogihara, M., Johnson, J., \& Huang, X., "Mining product reviews based on shallow dependency parsing", $32^{\text {nd }}$ International ACM SIGIR Conference on Research and Development in Information Retrieval, pp. 726-727, 2009.

[9] Ma, T., \& Wan, X., "Opinion target extraction in Chinese news comments", $23^{\text {rd }}$ International Conference on Computational Linguistics: Posters, Association for Computational Linguistics, pp. 782-790, 2010.

[10] Li, F., Han, C., Huang, M., Zhu, X., Xia, Y. J., Zhang, S., \& $\mathrm{Yu}, \mathrm{H}$., "Structure-aware review mining and summarization", $23^{\text {rd }}$ International Conference on Computational Linguistics, Association for Computational Linguistics, pp. 653-661, 2010.

[11] Jin, W., Ho, H. H., \& Srihari, R. K., "A novel lexicalized HMM-based learning framework for web opinion mining", $26^{\text {th }}$ ACM Annual International Conference on Machine Learning, pp. 465-472, 2009.

[12] Mei, Q., Ling, X., Wondra, M., Su, H., \& Zhai, C., "Topic sentiment mixture: modeling facets and opinions in weblogs", $16^{\text {th }}$ ACM International Conference on World Wide Web, pp. 171-180, 2007.

[13] Titov, I., \& McDonald, R., "A joint model of text and aspect ratings for sentiment summarization", ACL-08: HLT, 308$316,2008$.

[14] Zhao, W. X., Jiang, J., Yan, H., \& Li, X., "Jointly modeling aspects and opinions with a MaxEnt-LDA hybrid", Conference on Empirical Methods in Natural Language Processing, Association for Computational Linguistics, pp. 56-65, 2010.

[15] Mukherjee, A., \& Liu, B., "Modeling review comments", $50^{\text {th }}$ Annual Meeting Assoc. Comput. Linguistics, Jeju, Korea, pp. 320-329, 2012.

[16] Qiu, G., Liu, B., Bu, J., \& Chen, C., "Expanding domain sentiment lexicon through double propagation", IJCAI, vol. 9, pp. 1199-1204, 2009.

[17] Qiu, G., Liu, B., Bu, J., \& Chen, C., "Opinion word expansion and target extraction through double propagation", Computational linguistics, vol. 37 , no. 1, pp. 927, 2011.

[18] Liu, K., Xu, L., \& Zhao, J., "Opinion target extraction using word-based translation model", Joint Conference on Empirical Methods in Natural Language Processing and Computational Natural Language Learning, Association for Computational Linguistics, pp. 1346-1356, 2012.

[19] Kaur, A., \& Gupta, V., "A survey on sentiment analysis and opinion mining techniques", Journal of Emerging Technologies in Web Intelligence, vol. 5, no. 4, pp. 367-371, 2013. 
[20] Hasan, K. A., \& Rahman, M., "Sentiment detection from bangla text using contextual valency analysis", $17^{\text {th }}$ IEEE International Conference on Computer and Information Technology, pp. 292-295, 2014.

[21] Asghar, M. Z., \& Khan, A., "Sentiment classification through semantic orientation using SentiWordNet", Life Science Journal, vol. 11, no. 10, 2014.

[22] Ahmed, K., El Tazi, N., \& Hossny, A. H., "Sentiment analysis over social networks: an overview", IEEE International Conference on Systems, Man, and Cybernetics, pp. 2174-2179, 2015.

[23] Sri, R. J., \& Ajitha, P., "Survey of product reviews using sentiment analysis", International Journal of Science and Technology, 2016.

[24] Zhang, Y., Tang, F., Barolli, L., Yang, Y., \& Xu, W., "Jointly modeling multi-grain aspects and opinions for largescale online review", $31^{\text {st }}$ IEEE International Conference on Advanced Information Networking and Applications, pp. 570-577, 2017.

[25] Dubey, A., Gupta, A., Raturi, N., \& Saxena, P., "Item-Based Collaborative Filtering Using Sentiment Analysis of User Reviews", International Conference on Application of Computing and Communication Technologies, pp. 77-87, Springer, Singapore, 2018.

[26] Al-Ayyoub, M., Khamaiseh, A. A., Jararweh, Y., \& Al-Kabi, M. N., "A comprehensive survey of arabic sentiment analysis", Information processing \& management, vol. 56, no. 2, pp. 320-342, 2019.

[27] Gopi, A. P., Jyothi, R. N. S., Narayana, V. L., \& Sandeep, K. S., "Classification of tweets data based on polarity using improved RBF kernel of SVM", International Journal of Information Technology, pp. 1-16, 2020.

[28] Sankar, H., Subramaniyaswamy, V., Vijayakumar, V., Arun Kumar, S., Logesh, R., \& Umamakeswari, A. J. S. P., "Intelligent sentiment analysis approach using edge computing-based deep learning technique", Software: Practice and Experience, vol. 50, no. 5, pp. 645-657, 2020.

[29] Nielsen, M. A., "Neural networks and deep learning", vol. 2018, San Francisco, CA: Determination press, 2015.

[30] Cortes, C., \& Vapnik, V., "Support-vector networks", Machine learning, vol. 20, no. 3, pp. 273-297, 1995.

[31] Pontil, M., \& Verri, A., "Support vector machines for 3D object recognition", IEEE Transactions on Pattern Analysis and Machine Intelligence, vol. 20, no. 6, pp. 637-646, 1998.

[32] Yu, G.X., Ostrouchov, G., Geist, A., \& Samatova, N.F., “An SVM-based algorithm for identification of photosynthesisspecific genome features", IEEE Bioinformatics Conference, pp. 235-243, 2003.

[33] Joachims, T., "Text categorization with support vector machines: Learning with many relevant features", Springer Berlin Heidelberg, pp. 137-142, 1998.

[34] Mouthami, K., Devi, K.N. and Bhaskaran, V.M., "Sentiment analysis and classification based on textual reviews", International Conference on Information Communication and Embedded Systems, pp. 271-276, 2013. 